Article

\title{
A Dynamic Range Enhanced Readout Technique with a Two-Step TDC for High Speed Linear CMOS Image Sensors
}

\section{Zhiyuan Gao, Congjie Yang, Jiangtao $\mathrm{Xu}$ * and Kaiming Nie}

School of Electronic Information Engineering, Tianjin University, 92 Weijin Road, Nankai District, Tianjin 300072, China; E-Mails: gaozhiyuan@tju.edu.cn (Z.G.); yangcongjie@tju.edu.cn (C.Y.); nkaiming@tju.edu.cn (K.N.)

* Author to whom correspondence should be addressed; E-Mail: xujiangtao@tju.edu.cn; Tel./Fax: +86-22-2789-0832.

Academic Editor: Vittorio M. N. Passaro

Received: 31 August 2015 / Accepted: 2 November 2015 / Published: 6 November 2015

\begin{abstract}
This paper presents a dynamic range (DR) enhanced readout technique with a two-step time-to-digital converter (TDC) for high speed linear CMOS image sensors. A multi-capacitor and self-regulated capacitive trans-impedance amplifier (CTIA) structure is employed to extend the dynamic range. The gain of the CTIA is auto adjusted by switching different capacitors to the integration node asynchronously according to the output voltage. A column-parallel ADC based on a two-step TDC is utilized to improve the conversion rate. The conversion is divided into coarse phase and fine phase. An error calibration scheme is also proposed to correct quantization errors caused by propagation delay skew within $-T_{\text {clk }} \sim T_{\text {clk. }}$ A linear CMOS image sensor pixel array is designed in the $0.13 \mu \mathrm{m}$ CMOS process to verify this DR-enhanced high speed readout technique. The post simulation results indicate that the dynamic range of readout circuit is $99.02 \mathrm{~dB}$ and the ADC achieves $60.22 \mathrm{~dB}$ SNDR and $9.71 \mathrm{bit} \mathrm{ENOB}$ at a conversion rate of $2 \mathrm{MS} / \mathrm{s}$ after calibration, with $14.04 \mathrm{~dB}$ and 2.4 bit improvement, compared with SNDR and ENOB of that without calibration.
\end{abstract}

Keywords: CMOS image sensor; CTIA; wide dynamic range; two-step TDC; error calibration 


\section{Introduction}

Linear array image sensors obtain continuous images by scanning the target objects in one direction or in a loop, thus, they are usually used in industry detection, aerial photography, and satellite imaging. More and more applications require high speed scans to improve detection efficiency, and in some cases, the target objects are moving fast. Both conditions increase the demands of high line rate linear image sensors. Moreover, the target scene may contain high light and low light objects, and the dynamic range of the targets usually exceeds $90 \mathrm{~dB}$. However, the dynamic range of traditional readout circuits is limited to $60-70 \mathrm{~dB}[1-3]$. A wide dynamic range readout circuit is expected to cover at least a detecting range over $90 \mathrm{~dB}$ to capture all the possible information $[1,4]$. Therefore, the readout circuits of linear array image sensors require a wide detecting range and high speed readout.

For a linear CMOS image sensor (CIS), different types of readout techniques are used for various purposes, mainly including direct injection (DI), gate modulated injection (GMI), source follower per detector (SFD), and capacitor trans-impedance amplifier (CTIA) [5], etc. However, the integration linearity of DI and SFD gets significantly worse in dealing with wide range input signals. In GMI, the current gain and injection efficiency are sensitive to the threshold voltage change of MOS transistors, thus, readout circuits of different pixels may have large offset voltage [6]. Among these, CTIA can achieve good integration linearity and high injection efficiency, which makes it a popular readout circuit structure.

In order to fulfill the requirement of $90 \mathrm{~dB}$ photon dynamic range, a dynamic range extension technique has to be utilized. Various solutions for wide dynamic range CIS have been reported as CIS technology has developed. Some of them take advantage of improved pixel architectures, while others use novel readout methods. In general, they can be categorized into four main methods, i.e., logarithmic response, well capacity adjustment, multiple sampling, and saturation detection [7-9]. Well capacity adjustment and saturation detection are usually used in 4-T pixels. Logarithmic response and multiple sampling methods can be used with CTIA readout circuits. However, multiple sampling needs two or more times the readout operations than traditional pixels, which significantly limits the line rate. Logarithmic response is a simple and effective way, however, it suffers from fixed pattern noise when applied to APS. Therefore, a logarithmic-like response curve is created with a new CTIA-based dynamic range extension technique to achieve over $90 \mathrm{~dB}$ photon dynamic range.

High speed ADC is also necessary to achieve high line rates. Column-parallel ADC is the most widely used architecture in high speed linear imagers because it provides a better tradeoff among readout speed, silicon area and power consumption. Currently, three types of column-parallel ADCs have been used in CMOS image sensors: a successive approximation (SAR) ADC [10,11], a cyclic ADC [12], and a single-slope (SS) ADC [13,14]. Compared with SAR and cyclic ADCs, SS ADCs have many attractive features such as good linearity, simplicity of the analog circuits and resulting suitability for fine-pitched pixels. Moreover, they can ensure uniformity between columns and thus minimize column FPN. However, it may be difficult to simultaneously achieve both high-speed A/D conversion and high bit resolution because the required conversion time increases by a factor of $2^{\mathrm{N}}$ for N-bit resolution. To improve the conversion speed, some new methods have been reported: two-step single-slope ADC [15-17], multiple-ramp multiple-slope (MRMS) ADC [18], etc. In addition, further reduction in conversion time can be achieved by time-based SS ADC [19]. The input signal is converted to a 
time-domain representation using a ramp generator and a comparator and then the time domain information is quantized by a time-to-digital converter (TDC).

This paper presents a dynamic range enhanced, high speed readout technique consisting of a CTIA type readout circuit and a column-parallel SS ADC with two-step TDC. The proposed ADC has a significantly increased conversion rate compared with the conventional SS ADC [13,14], and costs less power and area than the multiple-ramp ADC [20].

The rest of this paper is organized as follows: Section 2 describes dynamic range enhanced column readout circuits as a preliminary to the following sections. Section 3 discusses the detailed design and operation of proposed $\mathrm{ADC}$, a calibration technique to mitigate the impact of mismatch in the two-step TDC is also presented. Section 4 presents the results, and Section 5 provides the conclusions.

\section{Dynamic Range Enhanced Readout Circuit}

The architecture of a linear image sensor with dynamic range enhanced readout circuits and two-step TDC based ADCs is shown in Figure 1. It consists of a linear photodiode array, dynamic range enhanced readout circuits, analog to digital converters, and peripheral circuits. The photodiode and readout circuit compose a pixel in the conventional sensors.

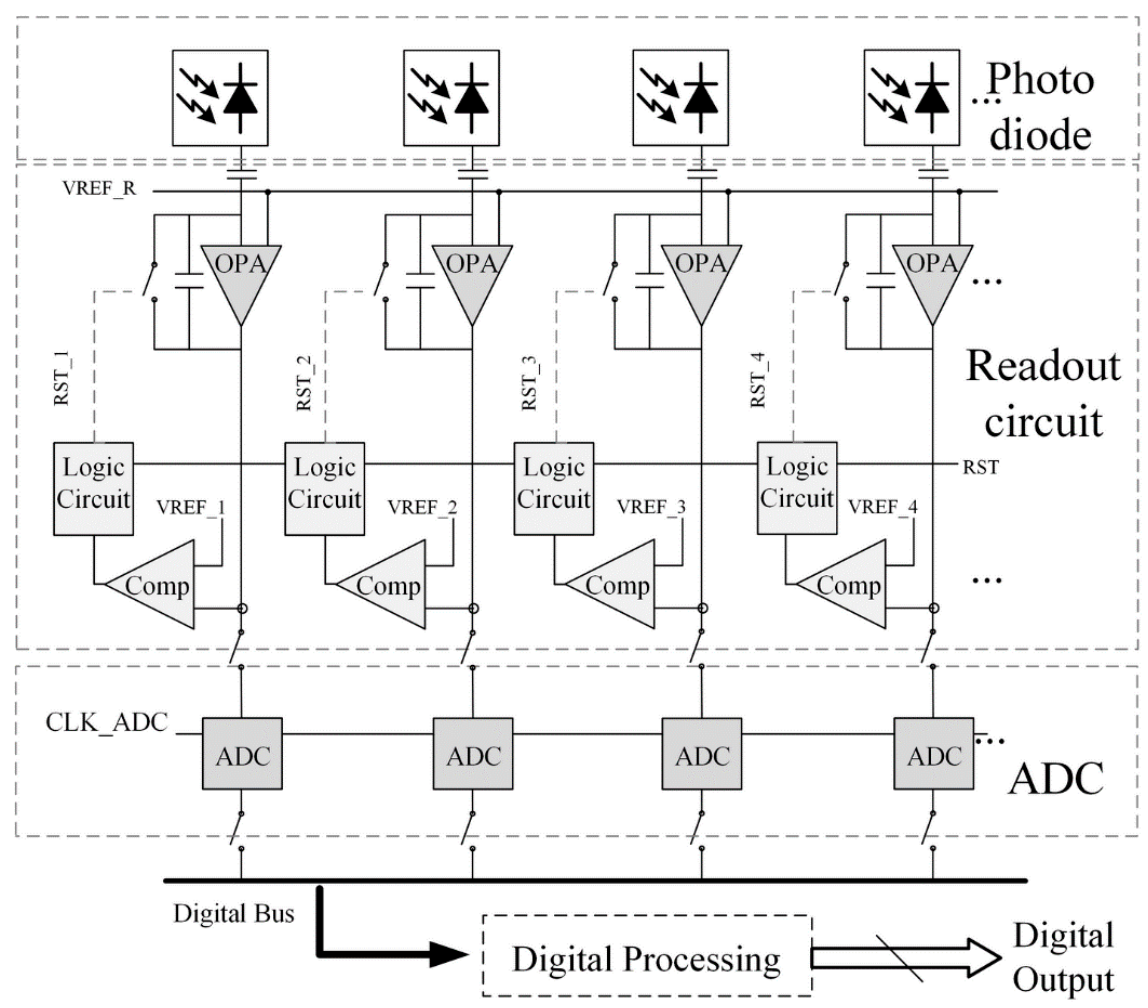

Figure 1. Block diagram of a linear image sensor.

\subsection{Capacitor Trans-Impedance Amplifier (CTIA)}

An improved CTIA structure is developed as the readout circuit in this paper due to its wide detection range, low noise and good integration linearity. Moreover, the non-destructive readout (NDR) can be realized with the CTIA structure. NDR mode is similar to the standard readout mode except that the output signals can be read out multiple times in a single readout integration time. The CTIA isolates the 
photodiode and the output signals by an amplifier, thus the readout operation does not have any effect on the photodiode.

The structure of CTIA is shown in Figure 2, where the integration capacitor is placed on the feedback loop of the amplifier with a reset switch to initialize the integration capacitor and reset the output to $V_{\text {ref. }}$ Additional integration capacitors $C_{\mathrm{g} 1}$ to $C_{\mathrm{g} 4}$ are connected to the circuit in parallel with $C_{\mathrm{int}}$. The left plates of $C_{\mathrm{g} 1}$ to $C_{\mathrm{g} 4}$ are connected to $V_{\mathrm{PD}} \mathrm{RST}$ in reset phase. A comparator at the output node is used to judge different reference voltages and control the amount of integrating capacitors accessing to CTIA at integration period. This CTIA-based structure has offset cancellation ability and dynamic range extension function.

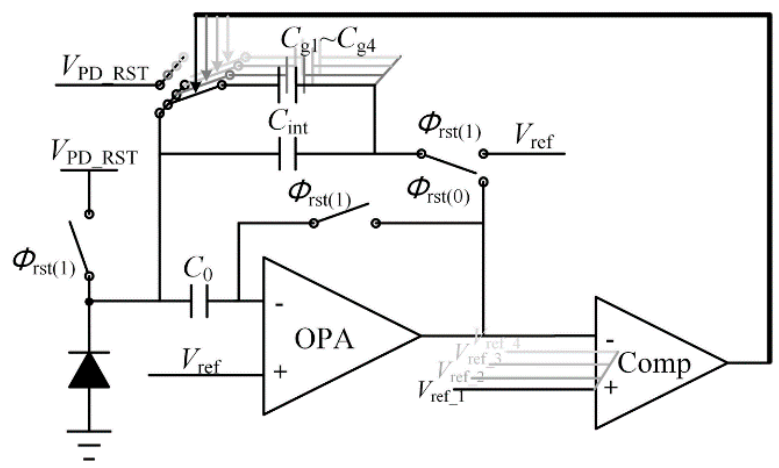

(a)

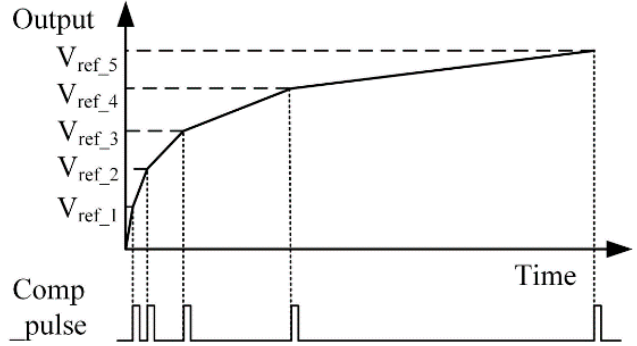

(b)

Figure 2. (a) The structure of CTIA; (b) The output curve of CTIA.

\subsubsection{Offset Cancellation}

Device mismatch and process variations may lead to offsets among pixels. A conventional CTIA-based pixel resets the photodiode by shorting the negative input and output of the OPA [21]. This method is simple, but an offset voltage induced by the OPA exists at the output, which is one of the sources of fixed pattern noise in pixel arrays. In this design, a reset transistor, an extra capacitor $C_{0}$ and a Single Pole Double Throw (SPDT) switch are adopted to eliminate the offsets at the output, also make the reset voltage of the photodiode flexible.

The switch sequence is shown in Figure 3 . During the reset period, $\Phi_{\mathrm{rst}(1)-(3)}$ signals are set as the logic high level to turn these switches on, as shown in Figure 3a. At the reset period, the photodiode is reset to $V_{\mathrm{PD}}$ RST, and the negative input and the output of the OPA is reset to $V_{\text {ref }}+V_{\text {os, }}$, where $V_{\text {os }}$ represents the offset voltage induced by the mismatches in the OPA. At the same time, the right plate of the integrating capacitor is always connected to $V_{\text {ref. }}$

The reset phase ends as $\Phi_{\text {rst(1) }}$ transits to its low level first as Figure $3 \mathrm{~b}$ shows. $K T / C$ noise is integrated on the photodiode, but it cannot propagate to the output as the right plates of $C_{\text {int }}$ and $C_{0}$ are still clamped. Then $\Phi_{\text {rst(2) }}$ transits to its low level as Figure $3 \mathrm{c}$ shows. The offset voltage is stored on $C_{0}$ while $V_{\text {out }}$ remains as $V_{\text {ref }}+V_{\text {os. }}$ When $\Phi_{\text {rst(3) }}$ is switched to the output of the OPA as shown in Figure 3d, because the charge stored on the integration capacitor $C_{\text {int }}$ and $C_{0}$ remains and they form a negative feedback, $V_{\text {out }}$

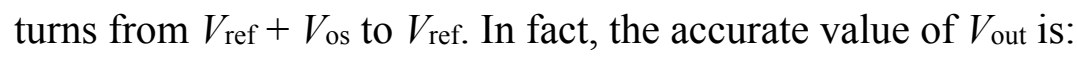

$$
V_{\text {out }}=V_{\text {ref }}-\frac{V_{\text {os }}}{A-1}
$$


where $A$ is the open-loop gain of the OPA in the CTIA structure. By considering the load effect of the capacitors, the frequency expression of the open-loop gain in a two-stage OPA is:

$$
A(s)=\frac{A_{0}}{\left(1+R_{\mathrm{I}} C_{\mathrm{I}} s\right) \cdot\left(1+R_{\mathrm{II}} C_{\mathrm{II}} s\right)}
$$

where $A_{0}$ is the open-loop DC gain, $R_{\mathrm{I}}\left(R_{\mathrm{II}}\right)$ and $C_{\mathrm{I}}\left(C_{\mathrm{II}}\right)$ are the resistance and capacitance to ground seen from the output of the first (second) stage. The capacitance load at output is derived as:

$$
\left\{\begin{array}{l}
C_{\mathrm{II}}(\text { reset phase })=C_{\mathrm{int}} \\
C_{\mathrm{II}}(\text { integration phase })=\frac{\left(\frac{C_{0} \cdot C_{\mathrm{in}}}{C_{0}+C_{\mathrm{in}}}+C_{\mathrm{pd}}\right) \cdot C_{\mathrm{int}}}{\left(\frac{C_{0} \cdot C_{\mathrm{in}}}{C_{0}+C_{\mathrm{in}}}+C_{\mathrm{pd}}\right)+C_{\mathrm{int}}} \approx \frac{C_{0} \cdot C_{\mathrm{in}}}{C_{0}+C_{\mathrm{in}}}+C_{\mathrm{pd}}
\end{array}\right.
$$

where $C_{\mathrm{in}}$ is the parasitic capacitance of input terminal, and $C_{\mathrm{pd}}$ is the parasitic capacitance of the photodiode. Since they are not large capacitance load, the OPA may achieve a good transient response characteristic easily. Furthermore, when the signal settles, the open-loop gain at low frequency is extremely large. Therefore, in Equation (1), the latter term is so small that it should be omitted, i.e., the offset voltage at output terminal is cancelled. Then the CTIA structure starts to integrate the photo current $I_{\mathrm{ph}}$ on the integration capacitor $C_{\text {int }}$ from $V_{\text {ref. }}$ Since the OPA is not ideal, its gain and the parasitic capacitance of the photodiode should be taken into consideration. Then the output voltage is derived as:

$$
V_{\text {out }}=\frac{A}{1+A}\left(V_{\text {ref }}+\frac{(A-1) I_{\mathrm{ph}} \cdot t}{(A-1) C_{\mathrm{int}}+C_{\mathrm{pd}}}\right)
$$

where $t$ is the integration time. From the equation above, if $A$ is large enough, the expression of $V_{\text {out }}$ can be simplified as:

$$
V_{\text {out }}=V_{\text {ref }}+I_{\mathrm{ph}} \cdot t / C_{\text {int }}
$$

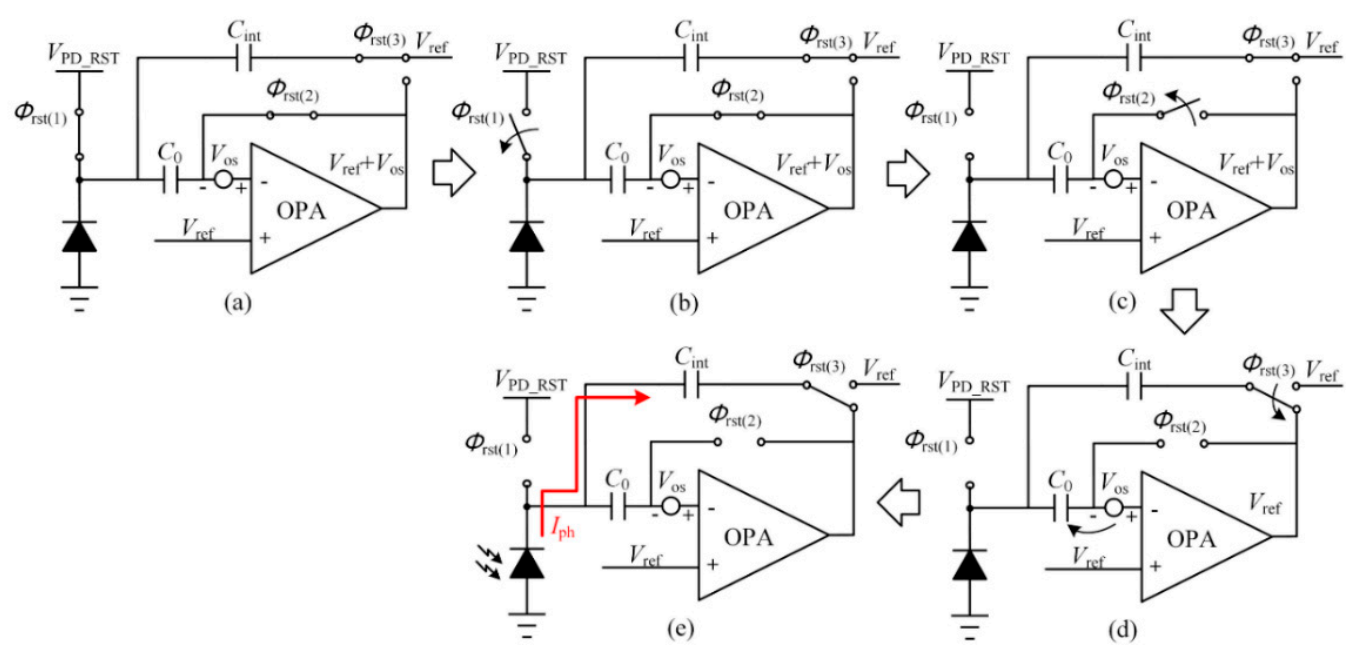

Figure 3. Switch sequence of the offset cancellation scheme. (a) Reset state; (b) $\phi_{\text {rst(1) }}$ is switched off; (c) $\phi_{\text {rst(2) }}$ is switched off; (d) $\phi_{\text {rst(3) }}$ is switched from $V_{\text {ref }}$ to the output terminal;

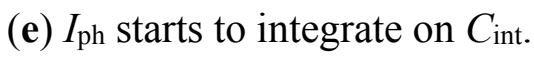




\subsubsection{Dynamic Range Extension}

In the conventional integration, the output increases linearly and saturates at $V_{\text {sat }}$ as Equation (5). CTIA operates as an integration circuit for photodiode. The photocurrent integrates on the capacitors connected across the input and output terminal which determines the sensitivity of integration. Sensitivity here is defined as the voltage variation under the conditions of 1 A photo current and $1 \mathrm{~s}$ integration time. Then the sensitivity could be derived from Equation (5) as:

$$
S=\frac{1}{C_{\mathrm{int}}}
$$

High sensitivity of integration can be achieved by using a small capacitance, and a large capacitance achieves the opposite [22]. A good sensitivity could improve the ability of low light detection and achieve low equivalent input noise, but it gets saturated under high light conditions. To address this issue, a multi-capacitor and self-regulation method is applied to the CTIA structure to achieve the wide dynamic range feature. Several capacitors, $C_{\mathrm{g} 1}$ to $C_{\mathrm{g} 4}$ in this design, are connected into the circuits in parallel with $C_{\text {int }}$ as additional integration capacitors. A reusable comparator is connected to the output terminal of the CTIA to monitor the signal voltage. The corresponding reference voltages, $V_{\text {refl }}$ to $V_{\text {ref5 }}$ are delivered by a MUX to the input terminal of the comparator. The sensitivity of the CTIA is automatically adjusted by switching different capacitors to the integration node asynchronously according to the output voltage. This method extends the dynamic range by compressing the photo response in the high light region as shown in Figure 2b. With a logarithmic-like photoresponse curve, the output under high light conditions would not get saturated compared to the traditional design with a single integration capacitor. Then both low and high light information is acquired.

The principle of this dynamic range extension approach is detailed as follows. When the reset phase

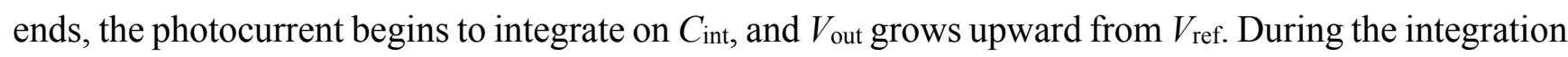
period, the comparator keeps monitoring $V_{\text {out, }}$, and the reference voltage is set as $V_{\text {refl }}$ at first. Once the signal voltage reaches the reference voltage $V_{\text {refl }}$, a short pulse is generated to switch $C_{\mathrm{g} 1}$ to the integration node. Thus, the integration capacitance increases and the sensitivity gets lower. After these operations, the reference voltage connected to the comparator is switched to the next predetermined

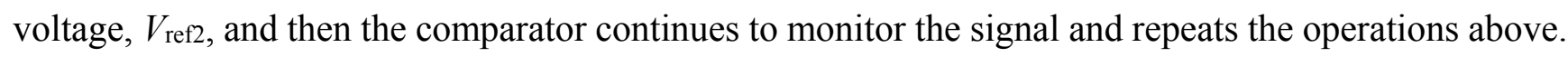
Assuming that light intensity is constant, then the output voltage of the CTIA is:

$$
V_{\text {out }}=I_{\text {ph }} \cdot \sum_{\mathrm{i}=1}^{\mathrm{n}} \frac{t_{\mathrm{i}}}{C_{\text {total_ } \mathrm{i}}}+V_{\text {ref }}
$$

where $t_{\mathrm{i}}$ is the integration time of $i$-th segment, and $\mathrm{C}_{\text {total }{ }_{-} \mathrm{i}}$ is the total capacitance used for photo current integration.

Thus, the sensitivity of each integration segment is:

$$
S_{\mathrm{i}}=\frac{1}{C_{\text {total } \_ \text {i }}}
$$

In the proposed design, four additional capacitors, $C_{\mathrm{g} 1}$ to $C_{\mathrm{g} 4}$, are to be connected to the integration node, and five reference voltages (including a saturation voltage) are alternately applied to the comparator. Then the photo response curve becomes a polyline with five segments before it gets saturated. The output 
swing in this design is from $1 \mathrm{~V}$ to $3 \mathrm{~V}$, which is divided into five segments, thus the reference voltages are $1.4,1.8,2.2,2.6$ and $3.0 \mathrm{~V}$, respectively.

\subsection{Readout Circuits Implementation}

In this design, a polyline type photoresponse curve with five segments is expected. The slope ratios of two adjacent segments are assumed to be equal. Then the slope ratio $k$ can be calculated by:

$$
k=1+\frac{C_{\mathrm{n}}}{\sum_{\mathrm{i}=0}^{\mathrm{n}-1} C_{\mathrm{i}}}(0 \leq n \leq 4)
$$

where $\mathrm{n}$ is the number of capacitors, $C_{\mathrm{n}}$ is the $n$-th capacitor that connected to the integration node. Each capacitance can be calculated by detection range and $k$. In this design, we define the maximum saturate current $I_{0}$ to be around $12 \mu \mathrm{A}$ within an integration time of $2 \mu \mathrm{s}$ as the target. The slope ratios of two adjacent segments are assumed to be equal, and the voltage swing of the readout circuit is $2 \mathrm{~V}$. According to these conditions, $\mathrm{k}$ is figured out to be 3.21. The initial capacitance is $400 \mathrm{fF}$, then four additional capacitors $C_{1}-C_{4}$ are $0.88 \mathrm{pF}, 2.84 \mathrm{pF}, 9.02 \mathrm{pF}$, and $29.03 \mathrm{pF}$. The total capacitance is $42.17 \mathrm{pF}$.

In order to capture clear image in fast scan application, the integration time in this design is as short as $2 \mu \mathrm{s}$. The maximum saturation current $I_{0}$ during this time is $12.3 \mu \mathrm{A}$, then the maximum charging current $I_{\text {total_max }}$ at the first integration segment is given by:

$$
I_{\text {total_max }}=I_{0} \times \frac{C_{\text {total }}}{C_{0}}
$$

From Equation (10), Itotal_max is around $1.3 \mathrm{~mA}$, i.e., the CTIA has to output $1.3 \mathrm{~mA}$ current to drive all these capacitors.

\subsubsection{OPA Design}

The OPA should be carefully designed to obtain such a large output current and reduce quiescent current as well. As shown in Figure 4, a class AB output stage is employed in OPA design. In order to achieve high speed detection, the amplifier may need to supply significant amounts of current to rapidly charge the load to the target accuracy. Therefore, an OPA with high gain, large output current, and low quiescent current is required. In this design, the OPA with class $\mathrm{AB}$ output stage achieves $102 \mathrm{~dB}$ gain and over $1.3 \mathrm{~mA}$ output current with a $130 \mu \mathrm{A}$ quiescent current.

\subsubsection{Logic Control and Comparator Design}

As shown in Figure 4, the control logic circuit is mainly composed of a pulse generator (PG), a reference voltage selector (RVS) and an integrating capacitor selector (ICS). PG is used to broaden the width of the reset pulse generated by the comparator when it is triggered. RVS delivers the corresponding reference voltage to the input of the comparator. ICS controls the amount of integrating capacitors accessing to CTIA for integration.

To acquire an accurate integrated voltage, the comparator should have sufficient gain, as well as the smallest propagation delay. The comparator uses three cascaded low-gain amplifiers as the preamplifier 
and a latch at the output, as shown in Figure 4. An offset cancellation technique is utilized to compensate the offsets of these amplifiers. As a result, the input offset of the comparator can be reduce to less than $0.5 \mathrm{LSB}$.

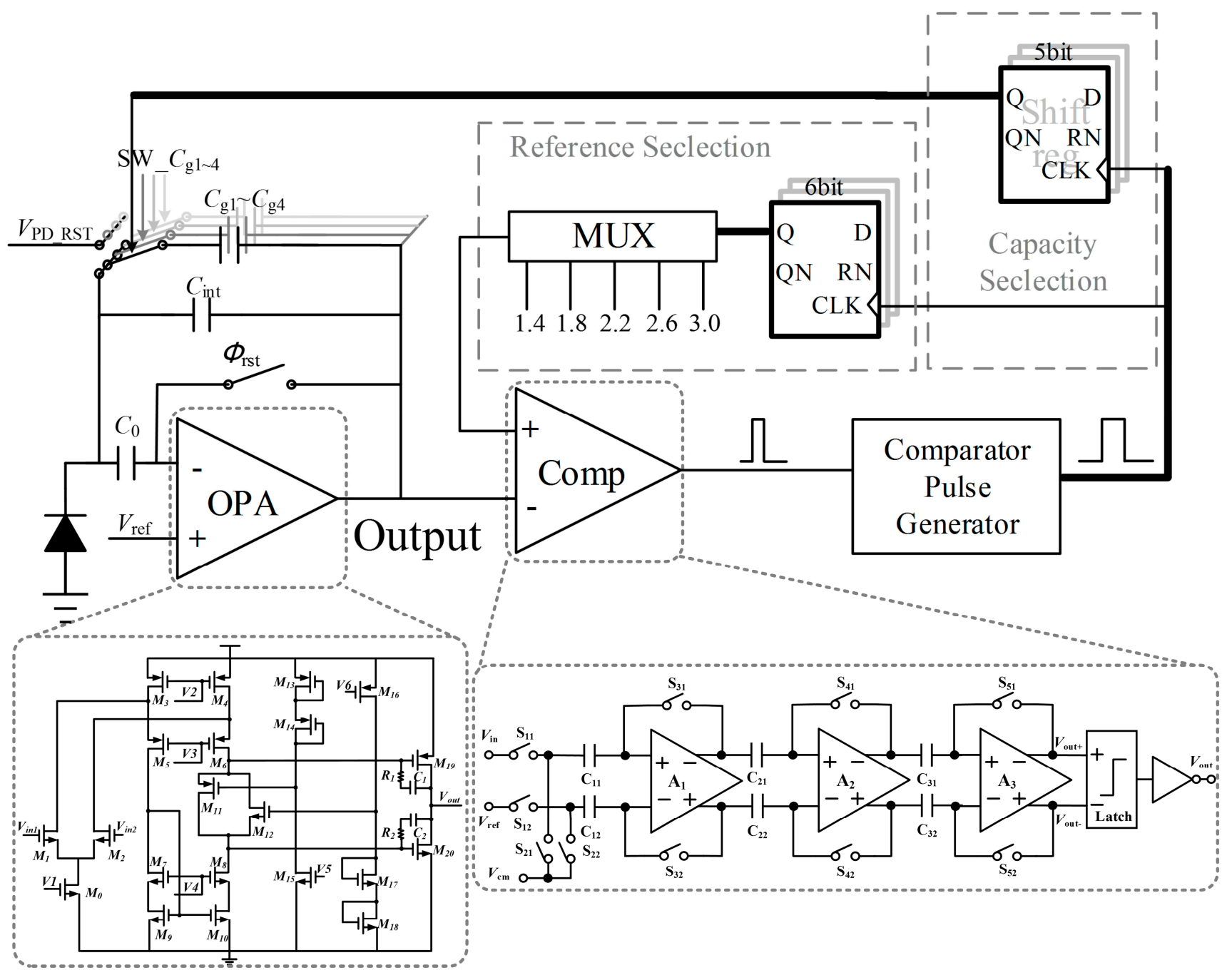

Figure 4. Circuit diagram of CTIA.

\section{Design of Column-Level ADC with Two-Step TDC}

The conventional column-level ADC usually operates at a relatively slow conversion speed. To achieve both wide dynamic range and high conversion rate, with low power consumption, a two-step TDC is utilized in this design.

\subsection{Operation Principle}

The basic concept of the proposed ADC consists of an analog-to-time conversion (ATC) and a parallel two-step TDC. The ATC transforms the sampled input voltage into a pulse signal whose width is linearly proportional to the input voltage. Then the width of the pulse signal is measured by the parallel two-step TDC, which yields a corresponding digital output. The n-bit conversion process of TDC is divided into $m$-bit coarse and $l$-bit fine conversions, where $n=m+l$. The coarse quantization is implemented by a counter which consumes little power, and the fine quantization is performed by a Vernier delay line 
(VDL) to obtain good accuracy [23]. By using this two-step architecture, the power consumption of TDC is reduced while maintaining large quantization range and fine resolution. The key challenges of this architecture are to achieve an efficient two-step TDC circuit architecture and ensuring the synchronization of two-step quantization.

\section{2. $A T C$}

Figure 5 shows the block diagrams and timing diagrams of the suggested linear ATC, which is composed of a sample-and-hold circuit, a ramp generator and a comparator. The linearity of ATC directly affects the ADC resolution [20]. In this design, OPA is used in the circuit to generate ramp voltage from $1 \mathrm{~V}$ to $3 \mathrm{~V}$. Compared with [20], the nonlinearity caused by the finite resistance of current source could be avoided as long as the gain is large enough.

The timing diagram of ATC is shown in Figure 5b. The switches, $S_{1}, S_{2}$ and $S_{3}$, are controlled by the start signal, which is generated by the timing module. When the start signal rises up to high level, $V_{\text {ramp }}$

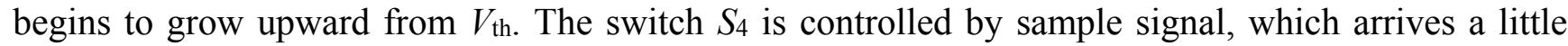
earlier than the start signal. When $V_{\text {sample }}$ and $V_{\text {ramp }}$ intersect, the comparator generates a pulse. Assuming that the ramp slope is constant, the time span between the start of the ramp and the comparator pulse is linearly proportional to the input voltage. The output signal of comparator is used as stop signal for TDC and the time interval $T_{\text {in }}$ which will be quantized is between the rising edge of start and stop signals.

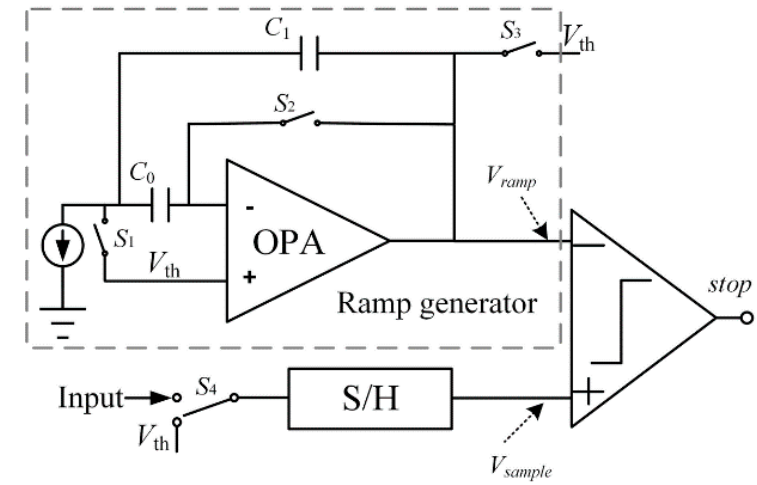

(a)

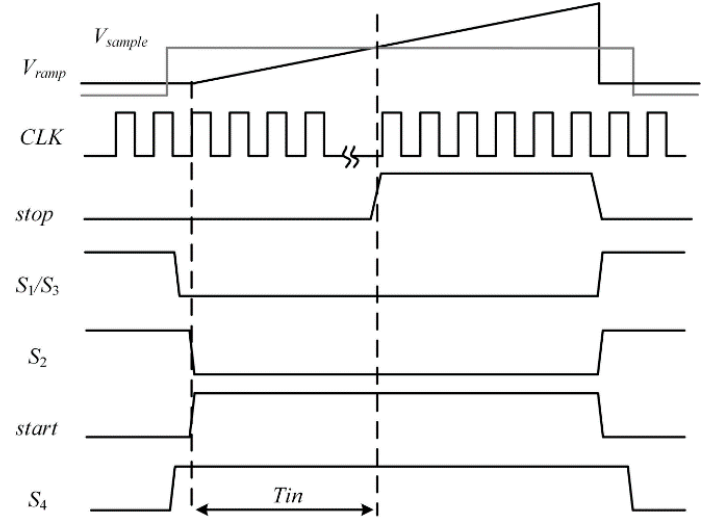

(b)

Figure 5. (a) Block diagram of ATC; (b) Timing diagram of ATC.

\section{3. $T D C$}

To achieve both energy efficiency and a large dynamic range, a two-step TDC combining a coarse counter and a VDL TDC is proposed. As shown in Figure 6, it is mainly composed of a VDL TDC, a coarse counter, a PLL that stabilizes clock frequency and a DLL to provide control voltages for VDL. A coarse counter quantizes the input time interval coarsely, and the residue is injected into a VDL TDC for fine quantization. Three more signals are introduced to describe the principle well: the start of fine quantization $S T_{1}$; the stop of fine quantization $S T_{2}$; and the enable signal of coarse quantization count_En. $S T_{1}$ is set by the rising edge of stop signal. $S T_{2}$ is set by the next rising edge of clock after the stop signal. count_En is set by start and reset by stop. Figure 7 shows the timing diagram of the two-step operation. A coarse counter measures the number of reference clock cycles while the count_En signal is 
high, i.e., it measures $T_{\mathrm{m}}$. The time interval between the rising edges of $S T_{1}$ and $S T_{2}$, defined as $T_{1}$, is measured by the fine TDC. As shown in Figure 7, the width of the input interval, defined as $T_{\text {in, }}$ is calculated by:

$$
T_{\mathrm{in}}=T_{\mathrm{m}}-T_{1}
$$

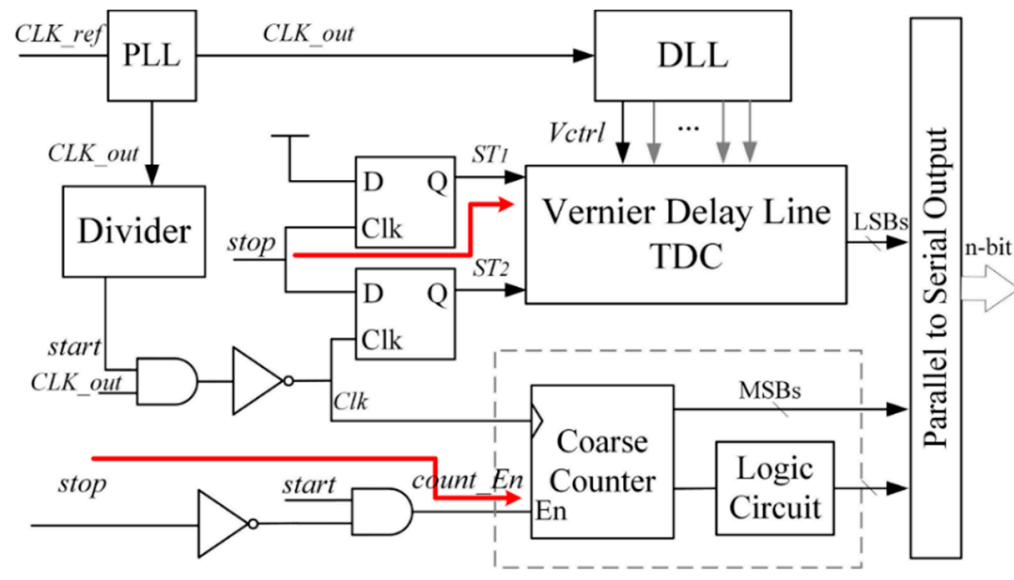

Figure 6. Block diagram of proposed TDC.

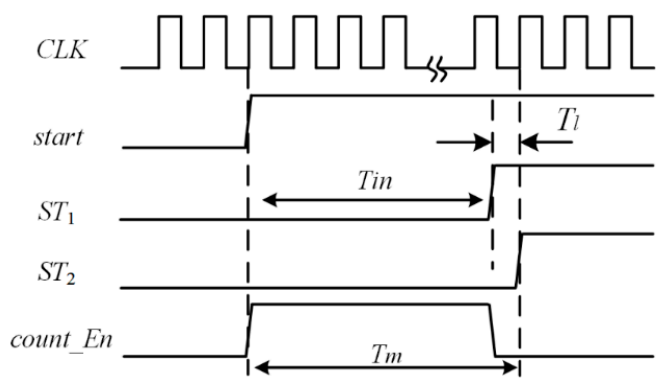

Figure 7. Timing diagram of proposed TDC.

The structure of VDL is shown in Figure 8, it consists of two delay-lines: One for the $S T_{1}$ and the other for $S T_{2}$. The up-line is composed of voltage-control delay elements and the down-line is composed of fixed delay elements. It is noted that the signal arrival time uncertainty grows with the length of the delay line. If more than 40 stages are implemented in the VDL, manufacture variations are the major sources of errors affecting the linearity. Sixteen stages are chosen for each delay line to quantize $T_{1}$.

The delay elements in the up-line have a delay $t_{\mathrm{d} 1}$ which is slightly longer than the delay $t_{\mathrm{d} 2}$ of the elements in the down-line. Thus, when the comparator output stop signal arrives, the $S T_{2}$ chases the $S T_{1}$. In each stage the delay difference is shortened by:

$$
\Delta t_{\mathrm{d}}=t_{\mathrm{d} 1}-t_{\mathrm{d} 2}
$$

The order status of two signals is detected by D flip-flops, and the output $Q_{1} Q_{2} \ldots Q_{\mathrm{n}}$ will be " $11 \ldots 00$ ". Assuming that the amount of " 1 " is $L$, then the interval of fine quantization is:

$$
\begin{aligned}
T_{1} & =L \times \Delta t_{\mathrm{d}} \\
& =L \times\left(t_{\mathrm{d} 1}-t_{\mathrm{d} 2}\right) \\
& =L \times T_{\mathrm{clk}} / 2^{N_{1}}
\end{aligned}
$$


Finally, this thermometer code will be translated to binary code and $l$-bits digital output is generated. Thus the TDC output can also be expressed as:

$$
\begin{aligned}
T_{\text {in }} & =T_{\mathrm{m}}-T_{1} \\
& =M \times T_{\mathrm{clk}}-L \times T_{\mathrm{ck}} / 2^{N_{1}}
\end{aligned}
$$

where $M$ is the coarse counter output.

However, the delay difference is uncertain as the digital delay elements depend on process variations, temperature, and supply voltage (PVT variations). The best way to stabilize the value of $\Delta t_{\mathrm{d}}$ against PVT variations and also provide calibration against process variations is to utilize Delay Lock Loop (DLL). DLL has two delay lines just the same as that in TDC as shown in Figure 8. Two similar signals only with one period time offset are injected into the delay lines, respectively. The output signals of the two delay lines are fed to a phase detector (PD) that compares the phase of these periodic signals. The phase detector generates a bias voltage $V_{\text {ctrl }}$ based on the phase difference to control the delay time, so that the delay of each element can be tuned by its control voltage $V_{\text {ctrl }}$, and eventually the TDC resolution is locked to: $\Delta t_{\mathrm{d}}=T_{\mathrm{clk}} / N$. A dummy DFF is also implemented in the DLL to balance the loading of the delay elements in the DFF.

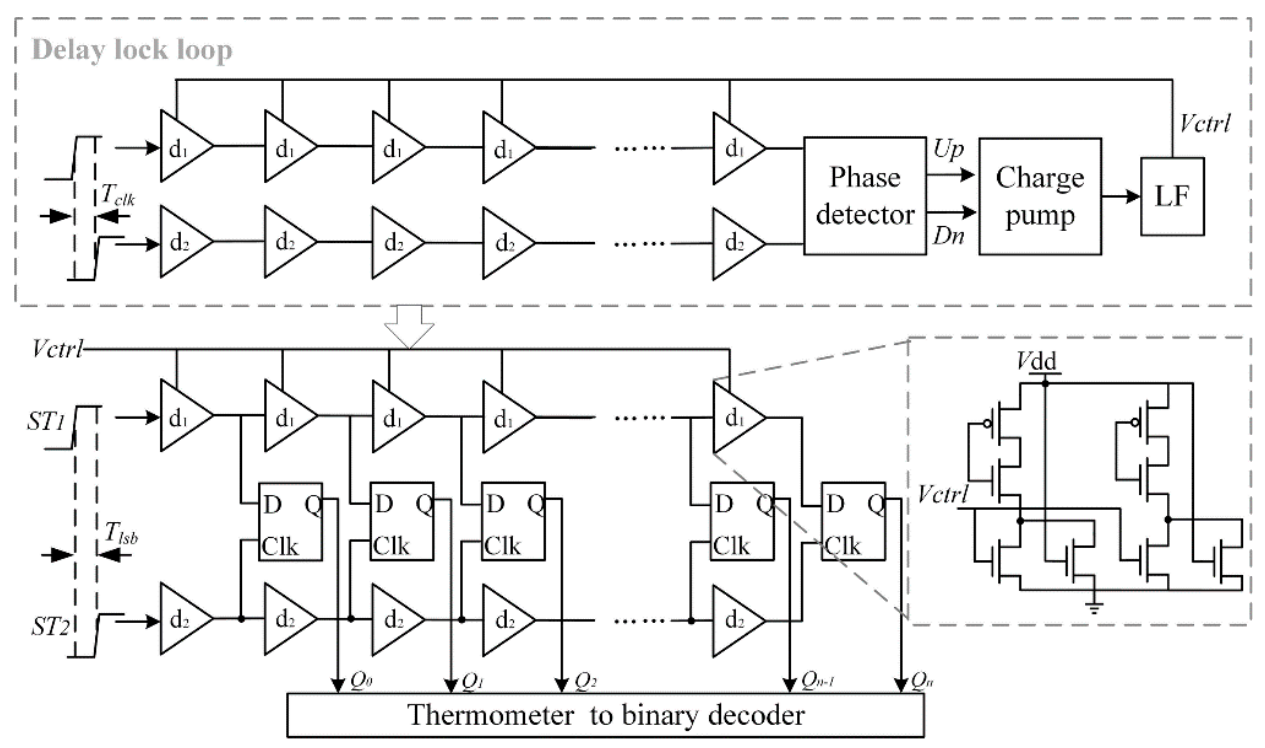

Figure 8. Block diagram of VDL and DLL.

\subsection{Quantization Error and Calibration}

For the proposed two-step TDC, the coarse quantization and fine quantization process the input signals in parallel and generate real-time outputs, the synchronization of input signals propagation should be guaranteed. This implies that the falling edge of stop signal of coarse quantization count_En must occur in the fine quantization cycle, or the quantization results may have a one-bit coarse quantized error. However, the delay time difference caused by device mismatches and process variations between different propagate paths make it impossible to align the coarse and fine quantization correctly. The quantization errors occur in two conditions, as shown in Figure 9. As depicted in Figure 9a, when the edge of count_En is coming before rising edge of the fine quantization clock cycle, the actual result 
of coarse quantization is 1LSB less than ideal result. On the contrary, when the edge of count_En is coming after the fine quantization clock cycle, the actual result of coarse quantization is 1LSB more than ideal result. Therefore, it causes quantization errors and seriously affects linearity.

Some latency can be compensated by additional elements in the propagate path, but additional delay elements also mean additional variability, additional power, and additional noise. In this paper, a calibration method is proposed to eliminate the quantization error with less additional circuits. As shown in Figure 9, the time latency between count_En and $S T_{1}$, is defined as $\Delta T_{\mathrm{p}}$, and the reasonable range of $\Delta T_{\mathrm{p}}$ is $-T_{\mathrm{clk}} \sim+T_{\mathrm{clk}}$.
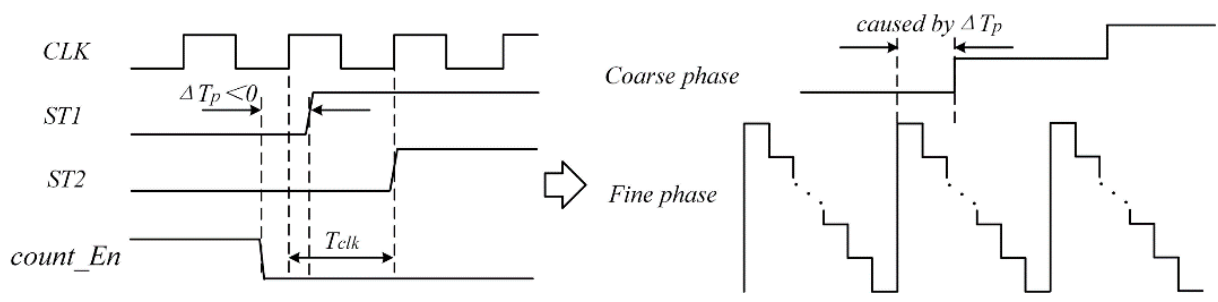

(a)
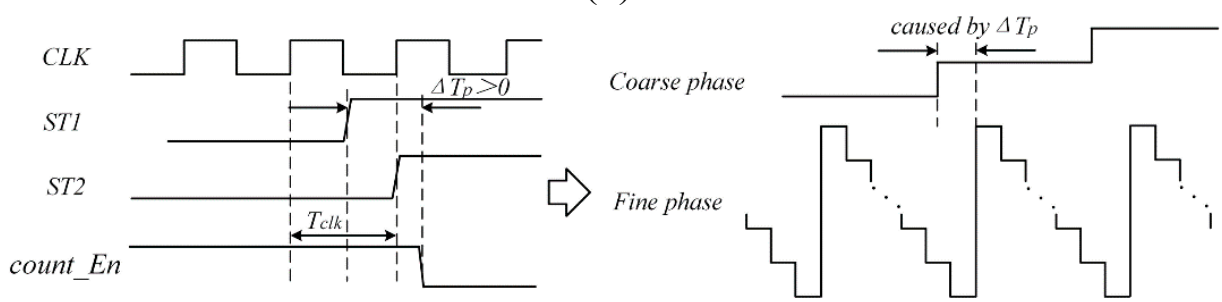

(b)

Figure 9. Timing diagrams of the TDC illustrating quantization error sources. (a) $\Delta T_{\mathrm{p}}<0$; (b) $\Delta T_{\mathrm{p}}>0$.

As depicted in Figure 10a, when $S T_{1}$ rises at the low level state of clock, the edge of count_En signal may appear in three positions $c l_{1}, c l_{2}, c l_{3}$, which indicate that the range of latency is $0 \sim+T_{\text {clk }}$, $-T_{\mathrm{clk}} / 2 \sim+T_{\mathrm{clk}} / 2,-T_{\mathrm{clk}} \sim 0$, respectively. When $S T_{1}$ rises at the high level state of clock, the edge of count_En signal may appear in three other positions $c h_{1}, c h_{2}, c h_{3}$, which indicate that the range of latency is $0 \sim+T_{\mathrm{clk}},-T_{\mathrm{clk}} / 2 \sim+T_{\mathrm{clk}} / 2,-T_{\mathrm{clk}} \sim 0$, respectively.

The proposed calibration circuit is illustrated in Figure 11, which is composed of a double input AND, a counter, a double edge-triggered D flip-flop, a falling edge-triggered D flip-flop, a triple input AND and a triple input NOR. The counter is modified as falling edge triggered, thus the last change in the LSB of the counter, $C_{0}$, could be caused by the edge of count_En signal. Compensation for coarse quantization result is decided by analyzing logic levels of clock and $S T_{1}$, when stop signal propagate to the coarse or fine quantization. The double edge-triggered DFF is used to obtain the state of count_En signal when $C_{0}$ changes. The falling edge-triggered DFF obtains the state of $S T_{1}$ when count_En falls. Furthermore, the MSB of fine quantitative output, denoted as $F_{\mathrm{n}}$, is utilized. In the case of $F_{\mathrm{n}}=0$, as shown in Figure 10a, which means $S T_{1}$ rises at the low level state of clock. If the falling edge of count_En signal appears in position $\mathrm{cl}_{3}$, which is at the high level state of the clock, the last change of $C_{0}$ comes from the falling edge of count_En, and the final output of DFF, $Q_{\mathrm{DFF}}=0$. In addition, $S T_{1}$ signal is in the high level when count_En changes, and it means the reverse output of DFF, $P_{\mathrm{DFF}}=0$. Then the $C_{-}$sub generated by "NOR" operation is high, thus it will subtract 1LSB from the coarse quantization output. Similarly, when the falling edge of count_En signal is in position $c l_{1}$ or $c l_{2}$, the correction bit $C_{-}$sub and 
$C$ _add are equal to 0 , which means no need for calibration. On the contrary, $\mathrm{ST}_{1}$ rises at the low level state of clock as shown in Figure 10b, in this case, $F_{\mathrm{n}}=1$. If the falling edge of count_En signal is in position $c h 1$, which is in the low level of the clock, the last change of $C_{0}$ comes from the falling edge of clock, and the final output of DFF, $Q_{\mathrm{DFF}}=1$. In addition, $S T_{1}$ signal is in the low level when count_En changes, and it means the reverse output of DFF, $P_{\mathrm{DFF}}=1$. Then the $C_{-}$add generated by "AND" operation is high, thus it will add 1LSB to the output. There is also no need for calibration when the falling edge of count_En signal is in position $\mathrm{ch}_{2}$ or $\mathrm{ch}_{3}$.

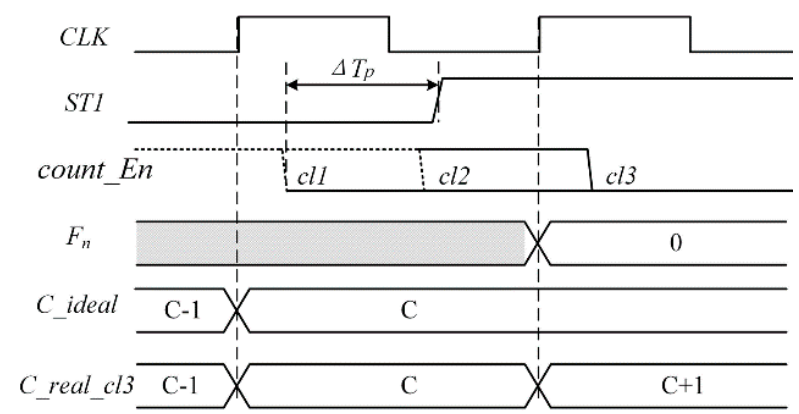

(a)

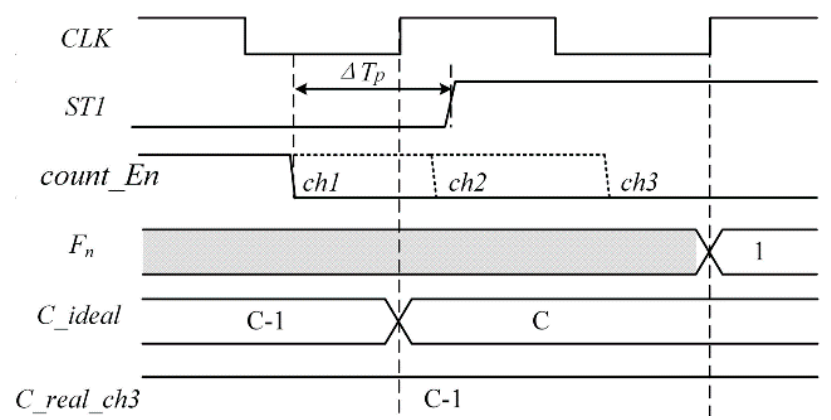

(b)

Figure 10. Timing diagram of the proposed calibration circuit. (a) $S T_{1}$ rises at the low level state of clock; (b) $S T_{1}$ rises at the high level state of clock ( $C_{-}$ideal denotes the ideal output of coarse quantization; $C_{-}$real denotes the actual output of coarse quantization).

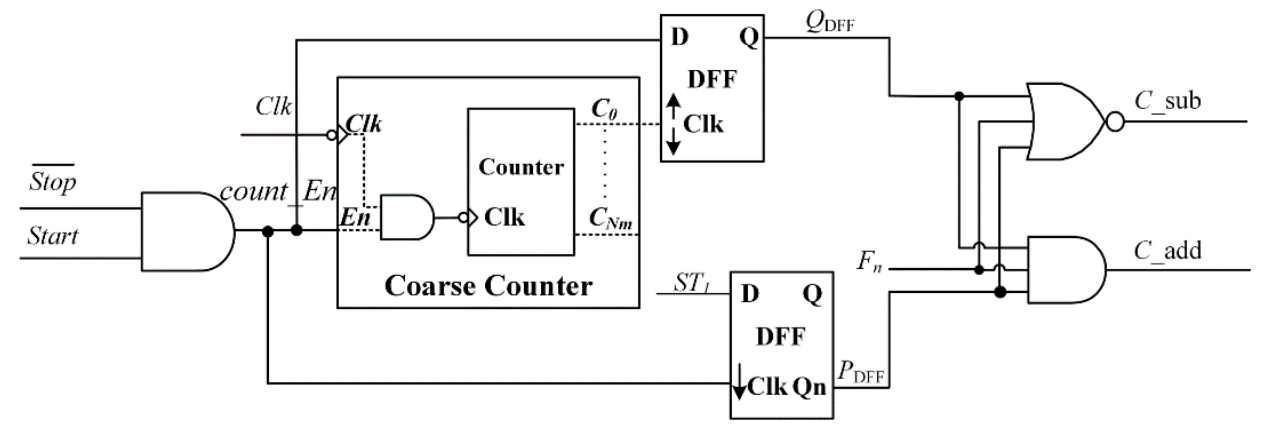

Figure 11. Block diagram of the proposed calibration circuit.

The proposed calibration method can effectively compensate the coarse quantization error caused by delay skew within $-T_{\mathrm{clk}} \sim+T_{\mathrm{clk}}$ with a slight increment of area and power consumption.

\section{Results}

A linear CMOS image sensor with $32 \times 1$ pixel array is designed in the $0.13 \mu \mathrm{m}$ CMOS process. The small-scale array is designed to prove the validity of readout circuits, and the pixel array could be extended, since the proposed readout circuits and ADCs are column wise designs. Figure 12 shows the layout of the linear image sensor. Each column readout circuit consumes $693 \mu \mathrm{W}$, and the ADC consumes $355 \mu \mathrm{W}$ power consumption provided by a $3.3 \mathrm{~V}$ supply for the analog circuits and a $1.5 \mathrm{~V}$ supply for the digital blocks. The following results are all from post-layout simulation. 


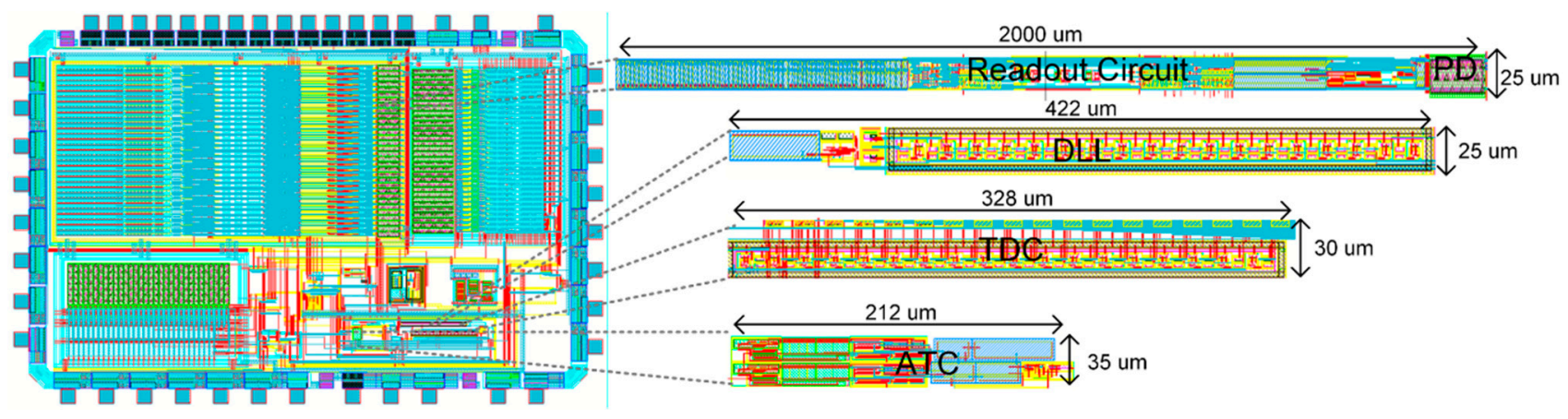

Figure 12. Layout of the linear image sensor.

\subsection{Readout Circuits}

The polyline type photocurrent response curve is shown in Figure 13. As the photocurrent increases, when the output voltage exceeds the threshold voltage each time, the sensitivity gets lower. Finally, the output curve presents as a polyline with five segments as expected. Figure 13 also shows the simulation of traditional CTIA with the same input current, and the integration capacitors are $400 \mathrm{fF}$ and $42 \mathrm{pF}$, respectively. When the integration capacitor is $400 \mathrm{fF}$, the CTIA saturates easily and the output signal will no longer keeps linearity with input current, and if the capacitor of $42 \mathrm{pF}$ is accessed to the circuit, the sensitivity is too low to distinguish the low light signals. The transient simulation of the pixel integration readout at different photocurrent is shown in Figure 14. The glitches on the output signal is mainly caused by two reasons: (1) The PD voltage slightly deviates from $V_{\mathrm{PD}}$ RST since the OPA is not ideal, which would make a difference between PD voltage and the voltage on the left plate of capacitors; (2) When the OPA outputs large current, the negative terminal of OPA deviates from $V_{\text {ref, }}$ which would also generate offset between PD voltage and the voltage on the left plate of capacitors; (3) The reset noise at the left plate of additional integration capacitors make its voltage various from $V_{\text {PD_RST, which }}$ contributes to the glitches and noise. Noise performance is also simulated, the total noise is $0.7 \mathrm{mV}$ including OPA and KT/C noise induced by capacitors and switches. Then the minimum photo current can be detected is $0.14 \mathrm{nA}$, and the non-saturated photo current is $12.5 \mu \mathrm{A}$. The dynamic range of this readout circuit is extended from $69.11 \mathrm{~dB}$ to $99.02 \mathrm{~dB}$.

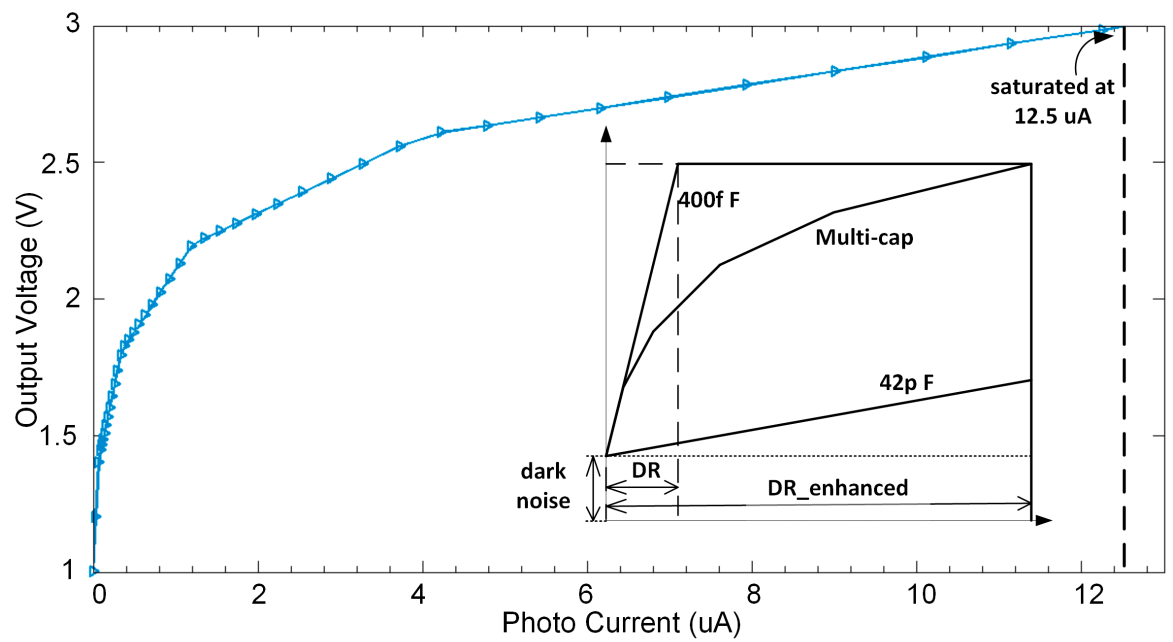

Figure 13. Output voltage versus photocurrent. The pixel saturates at around $12.5 \mu \mathrm{A}$. 


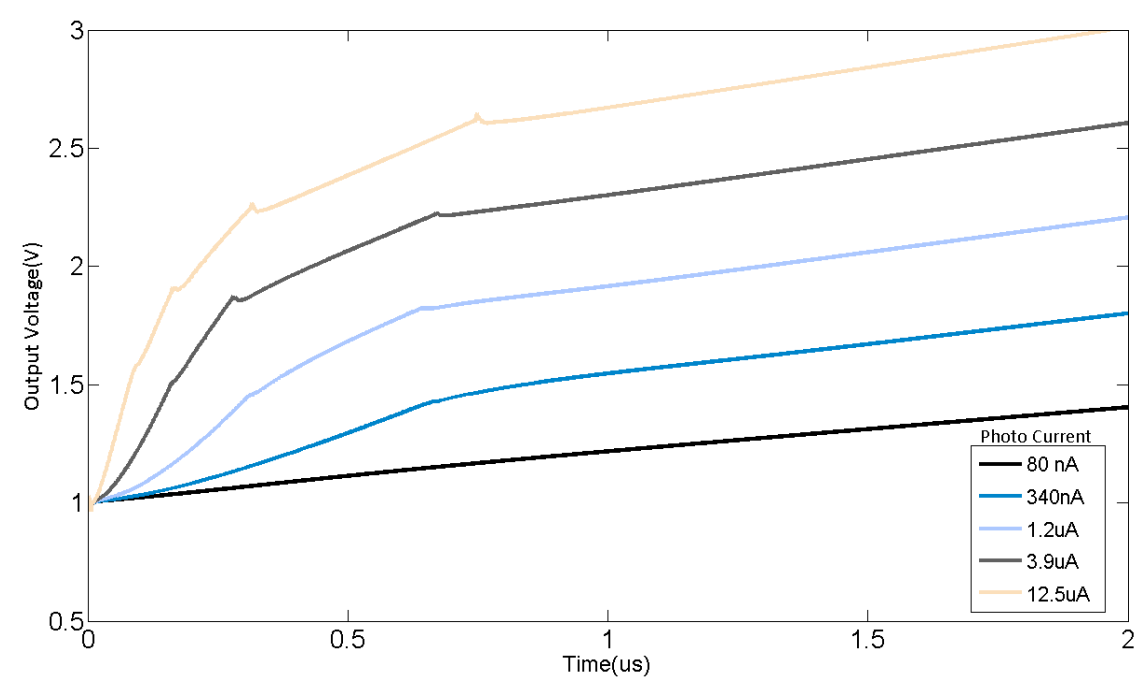

Figure 14. The transient simulation of the pixel integration.

\section{2. $A D C$}

Different delay skews are simulated by interpolating different dummy loads and delay cells to the propagation path. Simulated quantization error in negative cases $\left(\Delta T_{\mathrm{p}}<0\right)$ without calibration are illustrated in Figure 15.

(a)
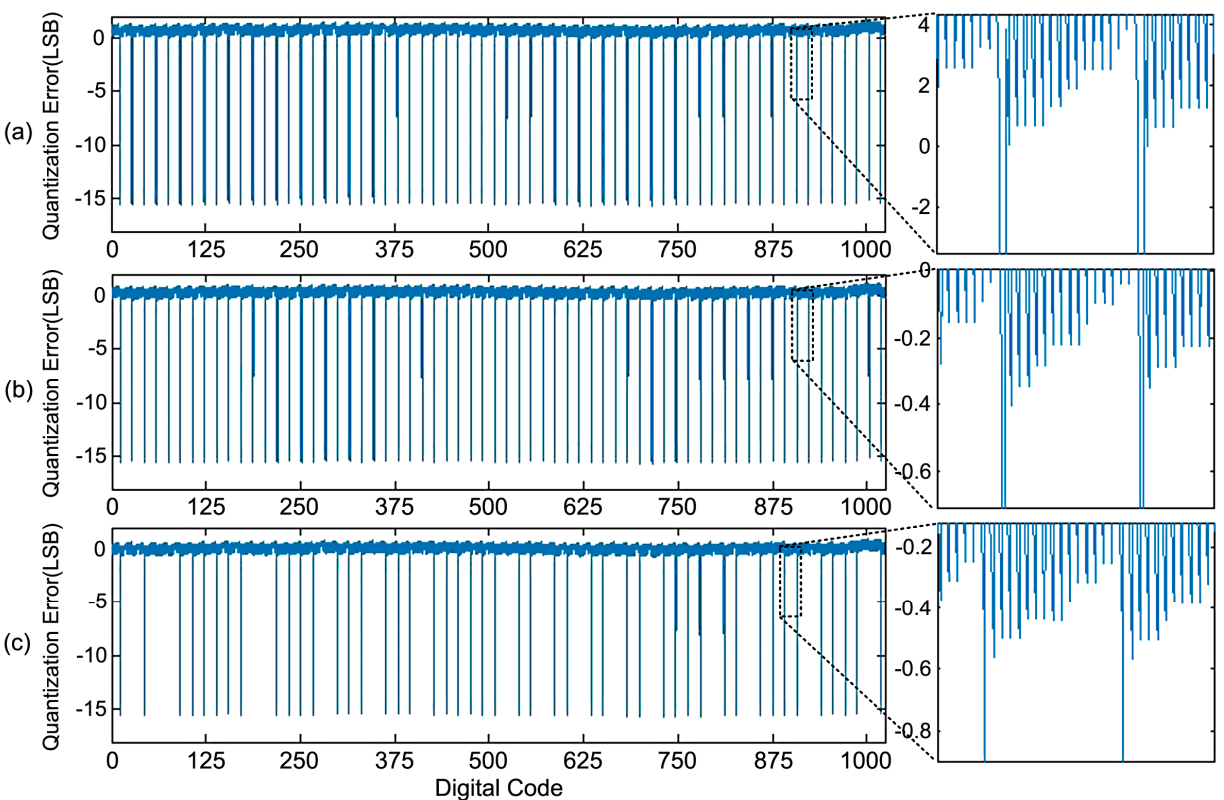

Figure 15. Quantization error without calibration. (a) $-T_{\mathrm{clk}}<\Delta T_{\mathrm{p}}<-T_{\mathrm{clk}} / 2$; (b) $-T_{\mathrm{clk}} / 2<\Delta T_{\mathrm{p}}<-100 \mathrm{ps}$; (c) $-100 \mathrm{ps}<\Delta T_{\mathrm{p}}<0$.

The quantization error gets better as the delay skew gets shorter, but even in Figure $15 \mathrm{c}$, i.e., when the delay skew is less than $100 \mathrm{ps}$, the quantization error still exists. The maximum deviation value is $+1.78 \mathrm{LSB} /-15.76 \mathrm{LSB}$ when $-100 \mathrm{ps}<\Delta T_{\mathrm{p}}<0$, which the following comparison is based on.

The maximum DNL and INL without calibration is $+15.90 /-15.36$ LSB and $+1.78 /-15.76$ LSB, which is easily obtained from the quantization error result. As shown in Figure 16, the DNL and INL drop to $+0.31 /-0.11 \mathrm{LSB}$ and $+0.25 /-0.51 \mathrm{LSB}$ with the proposed error correction. 

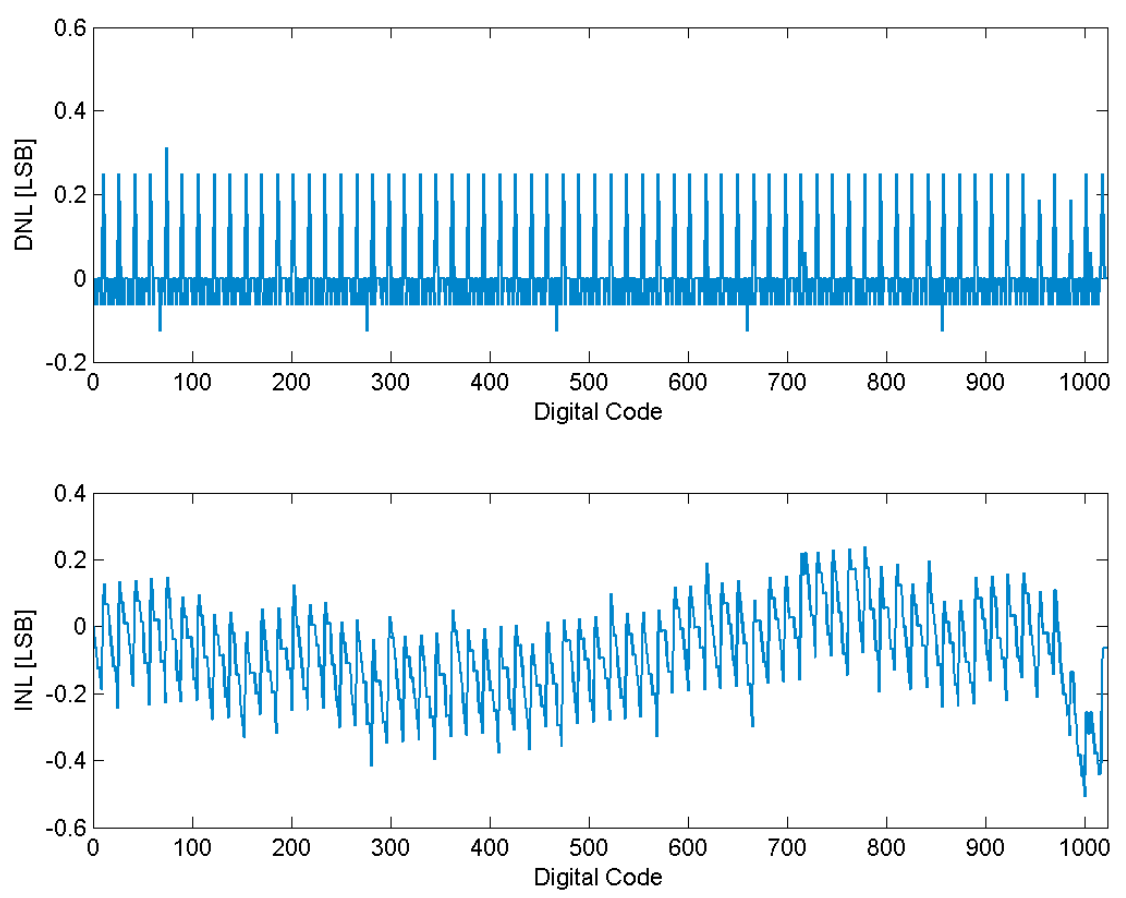

Figure 16. DNL and INL with calibration.

By applying a $470.7031 \mathrm{KHz}$ sinusoid input sampled at $2 \mathrm{MS} / \mathrm{s}$ in the simulation, the 1024-point FFT spectrum shows the ADC achieves 60.22 dB SNDR and $70.18 \mathrm{~dB}$ SFDR after calibration while it was limited to $46.18 \mathrm{~dB}$ SNDR and $64.17 \mathrm{~dB}$ SFDR before calibration, as illustrated in Figure 17. The ENOB measured is 7.37 bit without calibration, which is close to the coarse conversion accuracy. By applying the error calibration, the maximum ENOB has been improved to 9.71 bit. As the sinusoid input frequency decreases, the improvement effect is more remarkable. The comparison of characteristics is summarized in Table 1. The ADC performance has significantly improved with the proposed calibration technique.

Table 1. Simulated result comparison.

\begin{tabular}{ccc}
\hline & Without Calibration & With Calibration \\
\hline SFDR(@470.7031k), SNDR(@470.7031k) & $64.17 \mathrm{~dB} 46.18 \mathrm{~dB}$ & $70.18 \mathrm{~dB} 60.22 \mathrm{~dB}$ \\
ENOB(@470.7031k) & $7.31 \mathrm{bit}$ & $9.71 \mathrm{bit}$ \\
SFDR(@1.9531k), SNDR(@1.9531k) & $49.87 \mathrm{~dB} 39.38 \mathrm{~dB}$ & $69.59 \mathrm{~dB} 60.19 \mathrm{~dB}$ \\
ENOB(@1.9531k) & $6.25 \mathrm{bit}$ & $9.72 \mathrm{bit}$ \\
DNL(max) & $+15.90 /-15.36 \mathrm{LSB}$ & $+0.31 /-0.11 \mathrm{LSB}$ \\
$\mathrm{INL}(\max )$ & $+1.78 /-15.76 \mathrm{LSB}$ & $+0.25 /-0.51 \mathrm{LSB}$ \\
\hline
\end{tabular}

The linearity of the proposed image sensor is shown in Figure 18. The ADC output is extracted at different photo current, and the ideal code is calculated by Equation (7) at the same photo current sequence. An overall nonlinearity or INL as a function of photo current is also shown in this figure. The INL includes nonlinearity of the readout circuit and the column-ADC. The maximum INL is $+0.87 \% /-0.91 \%$ to the full scale of linear range. As shown in Figure 18, the nonlinearity in marked region is caused by charge injection effect when different capacitors are switched to the circuit. A good linearity could be obtained in every segment if the inflexion point is ignored.

The figure of merit (FOM) is defined as the following equation: 


$$
F O M_{A D C}=\text { Power } /\left(2^{\mathrm{ENOB}} \times F_{S}\right)
$$

where $F_{s}$ is $2 \mathrm{MS} / \mathrm{s}$, thus the $F O M_{A D C}$ of each column is $211 \mathrm{fJ} / \mathrm{step}$. Table 2 shows the performance comparisons of various column-parallel ADCs. The proposed ADC works in time-domain instead of voltage-domain, and thus the complexity and power consumption is significantly reduced. Compared with previous works, the proposed ADC achieves high conversion speed and the best FOM.

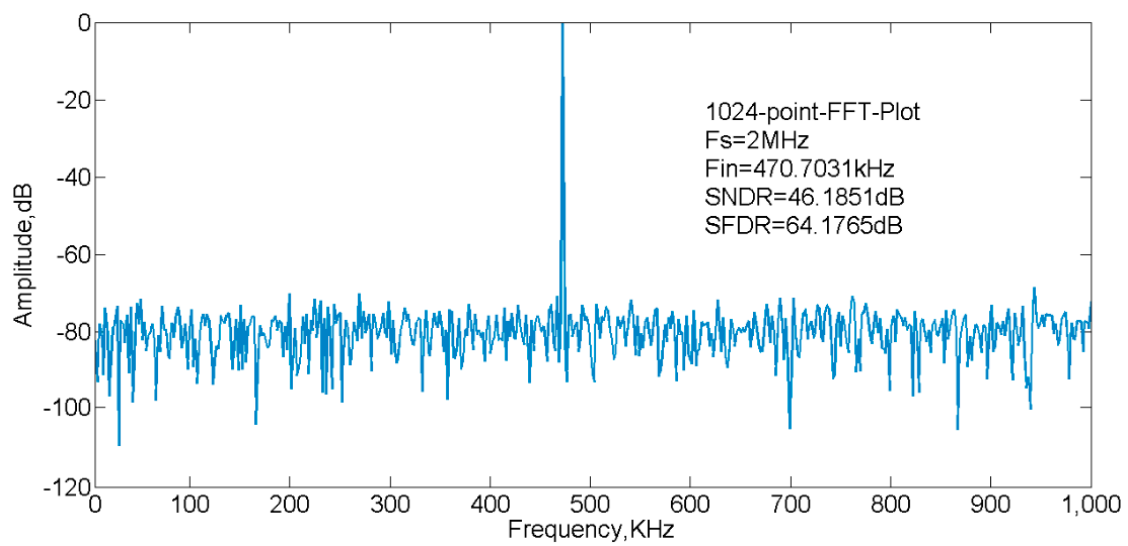

(a)

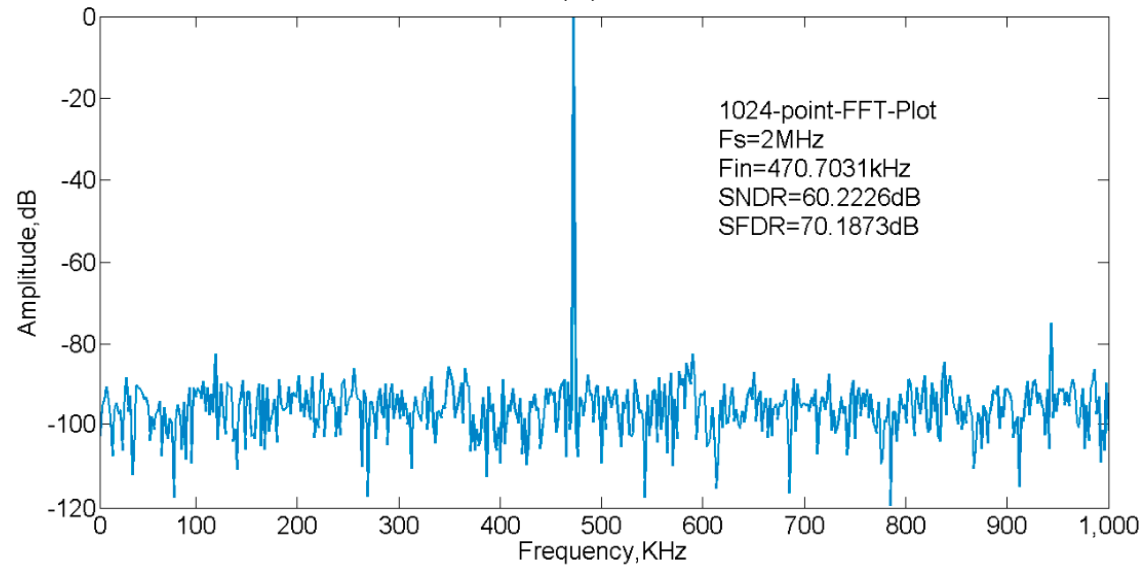

(b)

Figure 17. ADC output spectrum. (a) Without calibration; (b) With calibration.

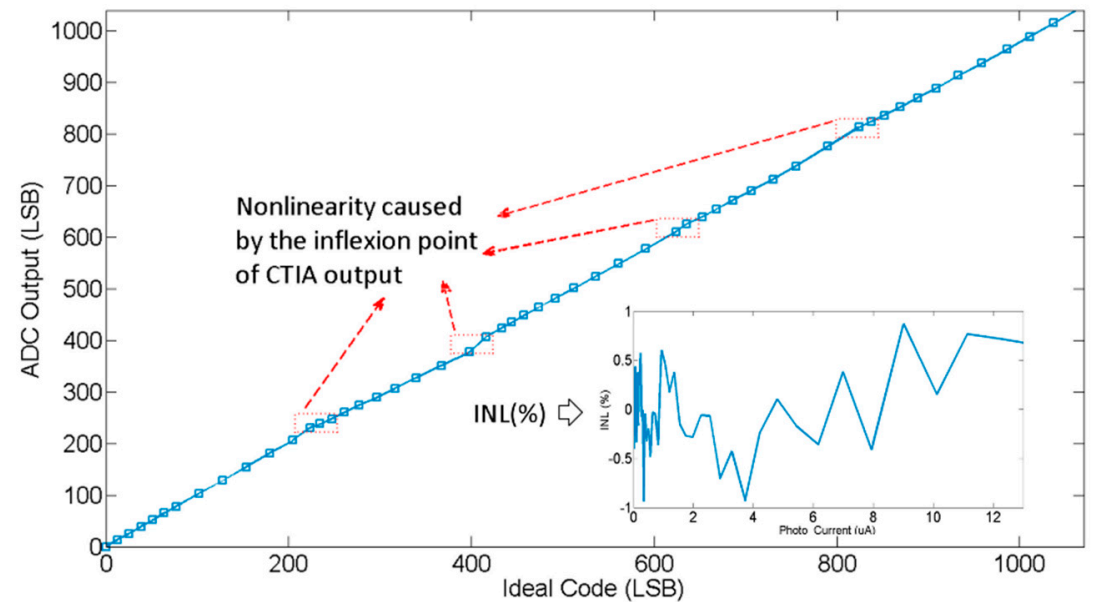

Figure 18. The linearity of the proposed image sensor 
Table 2. Comparison with other types of the column-parallel ADC.

\begin{tabular}{ccccccc}
\hline Reference & {$[\mathbf{1 0}]$} & {$[\mathbf{1 2}]$} & {$[\mathbf{1 4}]$} & {$[\mathbf{1 5}]$} & {$[\mathbf{1 8}]$} & This Work \\
\hline Technology & $0.18-\mu \mathrm{m}$ & $0.18-\mu \mathrm{m}$ & $0.18-\mu \mathrm{m}$ & $0.35-\mu \mathrm{m}$ & $0.25-\mu \mathrm{m}$ & $0.13-\mu \mathrm{m}$ \\
Architecture & SAR & Cyclic & Single Slope & Two-step SS & Multiple-ramp SS & SS With TDC \\
Resolution/bit & 8 & 13 & 12 & 10 & 10 & 10 \\
Conversion time/ $\mu \mathrm{s}$ & 1.2 & 6.4 & 9.5 & 4 & 16 & 0.5 \\
Power/ $\mu \mathrm{W}$ & 209 & $450 *$ & $166.7 *$ & 150 & 91 & 355 \\
FOM/fJ/step & 983.6 & 442.6 & 385.9 & 585.9 & 1138 & 211 \\
\hline \multicolumn{7}{c}{ * Values came from full-chip power consumption. }
\end{tabular}

All the sensor characteristics obtained from simulation results are listed in Table 3. The dynamic range is extended from $69.11 \mathrm{~dB}$ to $99.02 \mathrm{~dB}$ with nearly $30 \mathrm{~dB}$ improvement, and the ADC can effectively operate at $2 \mathrm{MS} / \mathrm{s}$ with a calibration method. Therefore, the proposed readout technique can fulfill both high speed and wide range detection features. The maximum line rate is $2 \mathrm{M}$ lines/s if the ADC is implemented as column circuits. Moreover, it can be configured accordingly, e.g., the line rate could be $500 \mathrm{~K}$ lines/s if one ADC is shared among four columns.

Table 3. Summary of the sensor characteristics.

\begin{tabular}{cc}
\hline Technology Photodiode Size & $\mathbf{0 . 1 3} \boldsymbol{\mu m} \mathbf{1 P 3 M} \mathbf{2 5} \boldsymbol{\mu m} \times \mathbf{1 0 0} \boldsymbol{\mu m}$ \\
\hline Supply Voltage & $3.3 \mathrm{~V} / 1.5 \mathrm{~V}$ \\
Line Rate & $2 \mathrm{M}$ \\
Sensitivity & $2.5 \mathrm{~V} / \mathrm{pA} \cdot \mathrm{s}(\max ) ; 0.025 \mathrm{~V} / \mathrm{pA} \cdot \mathrm{s}(\mathrm{min})$ \\
Noise Floor & $0.7 \mathrm{mV}$ \\
Dynamic Range & $99.02 \mathrm{~dB}$ \\
ADC resolution & $10 \mathrm{bit}$ \\
ADC ENOB & 9.72 bit (with calibration); 7.31 bit (without calibration) \\
Power Consumption & $1048 \mu$ W/column \\
\hline
\end{tabular}

\section{Conclusions}

A DR enhanced readout technique with two-step TDC for high speed linear CMOS image sensors is proposed in this paper. A multi-capacitor and self-regulated CTIA structure is proposed where the adjustable integration capacitors significantly extend the dynamic range. Moreover, a 10-bit column ADC based on two-step TDC with error calibration is proposed to improve the column conversion rate. The proposed ADC has a significantly increased conversion rate compared with the conventional SS $\mathrm{ADC}$, and requires less power and area than the multiple-ramp ADC. The calibration method can effectively compensate the quantization error caused by the propagation delay skew within $-T_{\mathrm{clk}} \sim+T_{\mathrm{clk}}$ without increasing the circuit complexity. Simulation results indicate that the readout circuits can achieve a dynamic range of $99.02 \mathrm{~dB}$ with nearly $30 \mathrm{~dB}$ improvement, and the ADC can achieve 9.71 bit ENOB at $2 \mathrm{MS} / \mathrm{s}$ with $355 \mu \mathrm{W}$ power consumption. The DNL/INL are significantly reduced down to $+0.31 /-0.11 \mathrm{LSB}$ and $+0.25 /-0.51 \mathrm{LSB}$ with calibration. Therefore, the proposed image sensor can fulfill the high speed and wide range detection features. 


\section{Acknowledgments}

Shilin Shen helped a lot in performing the simulation and revision of this manuscript. This work was supported by National Natural Science Foundation of China (61234003, 61274021).

\section{Author Contributions}

Zhiyuan Gao and Congjie Yang conceived the ideas and innovations, performed the simulation and analyzed the data; Jiangtao Xu provided supervision and guidance in this work; Kaiming Nie contributed a lot to the circuit design and layout; Zhiyuan Gao wrote the paper.

\section{Conflicts of Interest}

The authors declare no conflict of interest.

\section{References}

1. Srikantha, A.; Sidibé, D. Ghost detection and removal for high dynamic range images: Recent advances. Signal Process. Image Commun. 2012, 27, 650-662.

2. Spivak, A.; Benlenky, A.; Fish, A.; Yadid, P.O. Wide-Dynamic-Range CMOS Image Sensors - Comparative Performance Analysis. Electron Devices IEEE Trans. 2009, 56, 2446-2461.

3. Posch, C.; Matolin, D.; Wohlgenannt, R. A QVGA 143dB dynamic range asynchronous address-event PWM dynamic image sensor with lossless pixel-level video compression. In Proceeding of the 2010 IEEE International Conference on Solid-State Circuits Conference (ISSCC), San Francisco, CA, USA, 7-11 February 2010; pp. 400-401.

4. Kavusi, S.; Gamal, A.E. Quantitative study of high-dynamic-range image sensor architectures. Proc. SPIE 2004, 5301, pp. 264-275.

5. Ruan, A.W.; Shen, K.; Hu, B. Adjustable gain CTIA cell with variable integration time for IRFPA applications. In Proceeding of the International Conference on Communications, Circuits and Systems (ICCCAS), Milpitas, CA, USA, 23-25 July 2009; pp. 1066-1069.

6. Hsieh, C.C.; Wu, C.Y.; Sun, T.P.; Jih, F.W.; Cherng, Y.T. High-Performance CMOS Buffered Gate Modulation Input (BGMI) Readout Circuits for IRFPA. IEEE J. Solid-State Circuits 1998, 33, 1188-1198.

7. Cao, Y.; Pan, X.F.; Zhao, X.J.; Wu, H.S. An Analog Gamma Correction Scheme for High Dynamic Range CMOS Logarithmic Image Sensors. Sensors 2014, 14, 24132-24145.

8. Inkyu, B.; Byeungseok, Y.; Kyounghoon, Y. In-pixel calibration of temperature dependent FPN for a wide dynamic-range dual-capture CMOS image sensor. In Proceeding of the IEEE International Conference on Consumer Electronics (ISCE), JeJu Island, Korea, 22-25 June 2014; pp. 1-3.

9. Sasaki, M.; Mase, M.; Kawahito, S.; Tadokoro, Y. A wide-dynamic-range CMOS image sensor based on multiple short exposure-time readout with multiple-resolution column-parallel ADC. IEEE Sens. J. 2007, 7, 151-158.

10. Chen, D.G.; Fang, T.; Bermak, A. A Low-Power Pilot-DAC Based Column Parallel 8b SAR ADC with Forward Error Correction for CMOS Image Sensors. IEEE Trans. Circuits Syst. 2013, 60, 2572-2583. 
11. Shin, M.-S.; Kwon, O.-K. 14-bit two-step successive approximation ADC with calibration circuit for high-resolution CMOS imagers. Electron. Lett. 2011, 47, 790-791.

12. Seo, M.-W.; Suh, S.-H.; Iida, T.; Takasawa, T.; Isobe, K.; Watanabe, T.; Itoh, S.; Yasutomi, K.; Kawahito, S. A low-noise high intrascene dynamic range CMOS image sensor with a 13 to $19 \mathrm{~b}$ variable-resolution column-parallel folding-integration/cyclic ADC. Solid-State Circuits IEEE J. 2012, 47, 272-283.

13. Yeh, S.-F.; Hsieh, C.-C.; Cheng, C.-J.; Liu, C.-K. A novel single slope ADC design for wide dynamic range CMOS image sensors. In Proceeding of 2011 IEEE on Sensors, Limerick, Ireland, 28-31 October 2011; pp. 889-892.

14. Yoshihara, S.; Nitta, Y.; Kikuchi, M.; Koseki, K.; Ito, Y.; Inada, Y.; Kuramochi, S.; Wakabayashi, H.; Okano, M.; Kuriyama, H.; et al. A 1/ 1.8-ineh 6.4M Pixel 60 frames/s CMOS Image Sensor with Seamless Mode Change. IEEE J. Solid-State Circuits 2006, 41, 2998-3006.

15. Lim, S.; Lee, J.; Kim, D.; Han, G. A high-speed CMOS image sensor with column-parallel two-step single-slope ADCs. IEEE Trans. Electron Devices 2009, 56, 393-398.

16. Bae, J.; Kim, D.; Ham, S.; Chae, Y.; Song, M. A two-step a/d conversion and column self-calibration technique for low noise CMOS image sensors. Sensors 2014, 14, 11825-11843.

17. Lyu, T.; Yao, S.Y.; Nie, K.M.; Xu, J.T. A 12-Bit High-Speed Column-Parallel Two-Step Single-Slope ADC for CMOS Image Sensors. Sensors 2014, 14, 21603-21625.

18. Snoeij, M.F.; Theuwissen, A.J.P.; Makinwa, K.A.A.; Huijsing, J.H. Multiple-ramp column-parallel ADC architectures for CMOS image sensors. IEEE J. Solid-State Circuits 2007, 42, 2968-2977.

19. Muung, S.; Ikebe, M.; Motohisa, J.; Sano, E. Column parallel single-slope ADC with time to digital converter for CMOS imager. In Proceeding of the IEEE International Conference on Electronics, Circuits, and Systems (ICECS) 2010, Athens, Greece, 12-15 December 2010; pp. 863-866.

20. Naraghi, S.; Courcy, M.; Flynn, M.P. A 9-bit, $14 \mu \mathrm{W}$ and $0.06 \mathrm{~mm}$ Pulse Position Modulation ADC in $90 \mathrm{~nm}$ Digital CMOS. IEEE J. Solid-State Circuits 2010, 45, 1870-1880.

21. Murari, K.; Etienne-Cumimings, R.; Thakor, N.V.; Cauwenberghs, G. A CMOS In-Pixel CTIA High Sensitivity Fluorescence Imager. IEEE Trans. Biomed. Circuits Syst. 2011, 5, 449-458.

22. Kavusi, S.; Ghosh, K.; Abbas, E.G. Architectures for high Dynamic Range, High Speed Image Sensor Readout Circuits. In Proceeding of the International Conference on Very Large Scale Integration (VLSI), 2006 IFIP, Nice, France, 16-18 October 2006; pp. 36-41.

23. Dudek, P.; Szczepanski, S.; Hatfield, J.V. A high-resolution CMOS time-to-digital converter utilizing a Vernier delay line. IEEE J. Solid-State Circuits 2000, 35, 240-247.

(C) 2015 by the authors; licensee MDPI, Basel, Switzerland. This article is an open access article distributed under the terms and conditions of the Creative Commons Attribution license (http://creativecommons.org/licenses/by/4.0/). 